\title{
The trouble with ODE: polymerization during nanocrystal synthesis
}

\author{
Evert Dhaene, ${ }^{1}$ Jonas Billet, ${ }^{1}$ Ellie Bennett,${ }^{2}$ Isabel Van Driessche, ${ }^{1}$ Jonathan De Roo. $., 2,3 *$ \\ ${ }^{1}$ Department of Chemistry, Ghent University, Gent B-9000, Belgium \\ ${ }^{2}$ Department of Chemistry, Columbia University, New York, New York 10027, United States \\ ${ }^{3}$ Department of Chemistry, University of Basel, Basel CH-4058, Switzerland \\ * Corresponding author: Jonathan De Roo, Jonathan.DeRoo@unibas.ch
}

\begin{abstract}
1-octadecene is a widely used solvent for high temperature nanocrystal synthesis $(120-320$ $\left.{ }^{\circ} \mathrm{C}\right)$. Here, we show that 1 -octadecene spontaneously polymerizes under these conditions and the resulting poly(1-octadecene) has a comparable solubility and size to nanocrystals stabilized by hydrophobic ligands. Typical purification procedures (precipitation/redispersion cycles or size exclusion chromatography) fail to separate the poly(1-octadecene) impurity from the nanocrystal product. To avoid formation of poly(1-octadecene), we replace 1-octadecene with saturated, aliphatic solvents. Alternatively, the nanocrystals' native ligands are exchanged for polar ligands, leading to significant solubility differences between nanocrystals and poly(1octadecene), therefore allowing isolation of pure nanocrystals, free from polymer impurities. These results will help design superior syntheses and improve nanocrystal purity, an important factor in many applications.
\end{abstract}

\section{Keywords}

Quantum dots, Nanoparticles, Octadecene, Surface chemistry 


\section{Main text}

In the last 25 years, colloidal nanocrystals (NCs) have matured from an interesting physical phenomenon to a rich multidisciplinary field. ${ }^{1-6}$ While the chemical identity of the core determines most of the NC properties, the large surface-to-volume ratio makes surface chemistry a key enabler for applications. ${ }^{7-13}$ There are many synthetic techniques available for NCs, but solution phase synthesis stands out since it allows for crystallization at relatively low temperatures $\left(<400{ }^{\circ} \mathrm{C}\right)$ with high yield and versatility. ${ }^{14}$ An essential component of this approach is the solvent, which should be inert and have a high boiling point. In this respect, tri$n$-octylphosphine oxide (TOPO) is a commonly used, coordinating solvent for nanocrystal synthesis. However, commercial TOPO contains many impurities that bind to the NC surface and direct the crystal growth, yielding anisotropic structures. ${ }^{15-16}$ Even purified TOPO (obtained by recrystallization), ${ }^{17}$ can still decompose into by-products with high affinity for the surface. ${ }^{18}$ The resulting heterogeneous surface chemistry is disadvantageous as it complicates ligand exchange reactions. ${ }^{18}$ Non-coordinating 1-octadecene (ODE) is another frequently used solvent and seems to be plagued less by impurities. ${ }^{19-49}$ Apart from its role as solvent, ODE has often been used as reagent in NC synthesis since ODE's terminal alkene (vinyl) has inherent reactivity. For example, its redox properties have been harnessed to reduce elemental sulfur or selenium to its -II oxidation state, prior to incorporation in metal sulphide or selenide NCs. ${ }^{3,37}$, 43, 50-52 Furthermore, vinyl moieties can be polymerized by metallocene ${ }^{53-56}$ or inorganic ${ }^{57}$ catalysts. For this reason, Talapin et al. opted for saturated solvents and saturated ligands to avoid the gallium catalysed polymerization of ODE in the synthesis of GaAs NCs. ${ }^{58}$

Here, we study ODE's propensity to polymerize in the absence of catalysts, at temperatures relevant for $\mathrm{NC}$ synthesis. We find that $\mathrm{ODE}$ auto-polymerizes above $120^{\circ} \mathrm{C}$ with a temperature dependant molecular weight. Poly(ODE) is detected in the reaction product of five different NC syntheses; $\mathrm{ZnS}: \mathrm{Mn} / \mathrm{ZnS},{ }^{19} \mathrm{CuInS}_{2},{ }^{20} \mathrm{CdS},{ }^{14,21} \mathrm{TiO}_{2},{ }^{22}$ and $\mathrm{Fe}_{3} \mathrm{O}_{4}{ }^{23}$ We thus conclude that 
poly(ODE) is routinely contaminating NC production. To avoid the formation of poly(ODE), saturated solvents are used in those cases where ODE does not participate in the reaction. When ODE is necessary as reagent (and cannot be avoided), we show that a surface functionalization can provide a pathway to easily separate NCs from poly(ODE).

We heated ODE under argon to temperatures ranging from $100{ }^{\circ} \mathrm{C}$ to $320^{\circ} \mathrm{C}$. Whereas there is no reaction at $100{ }^{\circ} \mathrm{C}$, a colourless oil can be separated after 24 hours at $120^{\circ} \mathrm{C}$ (or higher) by the addition of acetone and subsequent centrifugation, a process not unlike the isolation of NCs from a crude reaction mixture. The ${ }^{1} \mathrm{H}$ NMR (nuclear magnetic resonance) spectrum of the oil confirms that an alkene resonance is absent (Figure S1), and the compound only consists of $\mathrm{CH}_{2}$ - and $-\mathrm{CH}_{3}$ moieties, with a relative intensity of $33: 3$, consistent with the polymerization of ODE into poly(ODE) (Figure 1A). To study the kinetics of the reaction, aliquots are taken at regular time intervals and analysed by quantitative ${ }^{1} \mathrm{H}$ NMR. The conversion of ODE to poly(ODE) is determined from the disappearance of the alkene resonance with respect to the methyl groups (Figure 1B). While the conversion becomes appreciable at $240{ }^{\circ} \mathrm{C}$, the conversion rate is dramatically high at $320{ }^{\circ} \mathrm{C}$ (the boiling point of ODE). After 24 hours, conversions of $0.64 \%, 6.58 \%, 24.01 \%$, and $88.51 \%$ are reached at $120,160,240$, and $320{ }^{\circ} \mathrm{C}$ respectively. After precipitation with acetone and purification, a yield by mass of respectively $0.1 \%, 3.4 \%, 14.8 \%$, and $66.3 \%$ is obtained. We also assessed the recovered yield (at $240{ }^{\circ} \mathrm{C}$ ) using other common non-solvents; ethanol (15.61\%), isopropanol (10.04\%), methyl acetate $(9.22 \%)$. We thus conclude that poly(ODE) has a higher solubility in isopropanol and methyl acetate, while the solubility in ethanol is similar to that in acetone. Acetonitrile and methanol were also considered but, giving their high polarity, formed a biphasic system with ODE.

Poly(ODE), synthesized at various temperatures, is subsequently analysed by size exclusion chromatography (SEC), revealing that the molecular weight is inversely related to the reaction temperature (Figure 1C). This indicates that the reaction is thermodynamically controlled. ${ }^{59}$ At 
higher reaction temperatures, the entropic factor dominates the free energy, thus favouring polymer chains with a lower molecular weight. Note that similar molecular weights are obtained using ODE which was either vacuum distilled or simply degassed (Figure S2). In addition to SEC, 2D diffusion ordered spectroscopy (DOSY) NMR has also been established as a technique to determine the molecular weight of polymers. ${ }^{60}$ DOSY organizes NMR resonances according to their diffusion coefficient, which is related to their solvodynamic size. Indeed, 2D DOSY NMR of the poly(ODE), synthesized at $240{ }^{\circ} \mathrm{C}$, yields a lower diffusion coefficient $\left(265 \mu \mathrm{m}^{2} / \mathrm{s}\right)$ compared to the parent ODE (Figure 1D). Using the Stokes-Einstein relation, we calculate a corresponding solvodynamic diameter of about $3.0 \mathrm{~nm}$.

A
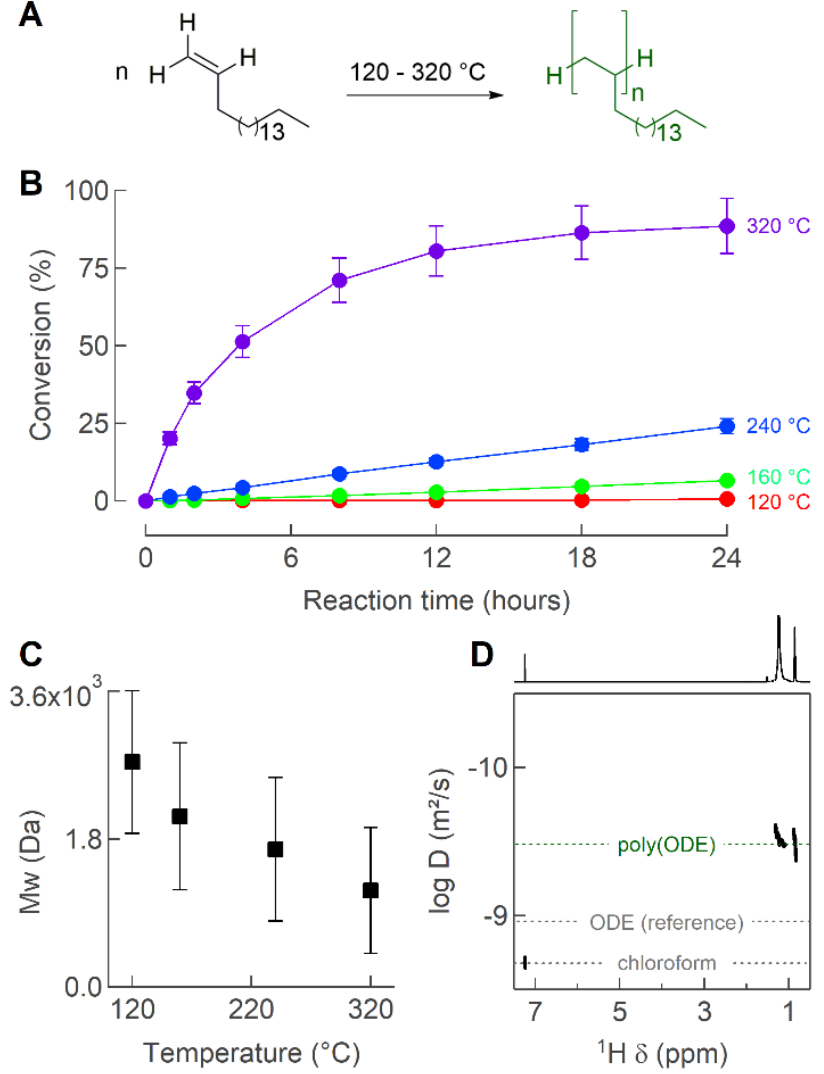

Figure 1. (A) Poly $(\mathrm{ODE})$ is obtained at temperatures between 120 and $320^{\circ} \mathrm{C}$. (B) The conversion kinetics were determined from the disappearance of the alkene resonance in quantitative ${ }^{1} \mathrm{H}$ NMR spectra of $10 \mu \mathrm{L}$ aliquots in $\mathrm{CDCl}_{3}$. (C) The molecular weight of poly(ODE), measured by SEC in tetrahydrofuran, with their standard deviation calculated from the average molecular weight and dispersity $\left(\bigoplus=1+\frac{\sigma^{2}}{\mu^{2}}\right)$, is inversely correlated to the reaction temperature. (D) $2 \mathrm{D}$ DOSY NMR in $\mathrm{CDCl}_{3}$ of poly(ODE) formed at $240{ }^{\circ} \mathrm{C}$ after 24 hours. The diffusion coefficient of 1-octadecene is added as a reference point (Figure S3). 
Since the polymerization of ODE proceeds without catalyst and is controlled by temperature and time, poly(ODE) is most likely formed in many NC syntheses. To demonstrate this, we analysed the reaction products of a broad range of NC syntheses, including core, core-shell and doped NCs: $\mathrm{ZnS}: \mathrm{Mn} / \mathrm{ZnS},{ }^{19} \mathrm{CuInS}_{2},{ }^{20} \mathrm{CdS},{ }^{14,21} \mathrm{TiO}_{2},{ }^{22}$ and $\mathrm{Fe}_{3} \mathrm{O}_{4} \cdot{ }^{23}$ These NC syntheses are well-established and the synthetic protocols are widely used. Based solely on the duration and temperature of the reaction (absent any metal catalysis), we expect a conversion to poly(ODE) of $0.72 \%, 0.43 \%, 2.37 \%, 2.45 \%$, and $3.28 \%$ for the different NC syntheses respectively. The NCs were purified using acetone as non-solvent and TEM analysis confirmed the successful synthesis of the NCs (Figure 2). However, the ${ }^{1} \mathrm{H}$ NMR spectra of the purified nanocrystals reveal that the integrals of the resonances are not consistent with the expected ligand. Consistently, the integrals for the $-\mathrm{CH}_{2}-$ and $-\mathrm{CH}_{3}$ resonances were too high, indicating an aliphatic impurity.

It is challenging to unambiguously confirm the presence of the polymer because of (i) the difficulty in physically separating the hydrophobic NCs from the hydrophobic polymer, and (ii) the spectral overlap between the aliphatic NMR resonances of the ligands and the polymer. Again, we use DOSY NMR to virtually separate the resonances according to their diffusion constant (Figure 2). In every nanocrystal sample we retrieved two sets of resonances, each diffusing with a different speed, one corresponding to the colloidal NCs and one to the poly(ODE). The ligand-capped NCs are larger in size than poly(ODE), and therefore have a lower diffusion coefficient. Note that the case of $\mathrm{Fe}_{3} \mathrm{O}_{4} \mathrm{NCs}$ is special since the NCs are magnetic, thus prohibiting direct NMR analysis. To circumvent this limitation, we exchanged the native oleate ligand for trifluoroacetic acid, causing the $\mathrm{Fe}_{3} \mathrm{O}_{4} \mathrm{NCs}$ to precipitate. The exchange is driven by a difference in $\mathrm{pKa}$ (trifluoroacetic acid is a stronger acid compared to oleic acid). ${ }^{7}$ The small trifluoroacetate ligand do not support colloidal stability and as a result, the NCs precipitate. ${ }^{61-62}$ The supernatant contains the free, unbound oleic acid (with a high 
diffusion coefficient) and the poly(ODE) (Figure 2). In the case of $\mathrm{TiO}_{2} \mathrm{NCs}$, the production of poly(ODE) is most noticeable compared to the other syntheses. This is most likely due to the catalytic properties of titanium halide precursors with respect to the polymerization of olefins. ${ }^{57}$

Overall, we conclude that poly(ODE) is present in all studied NC samples.

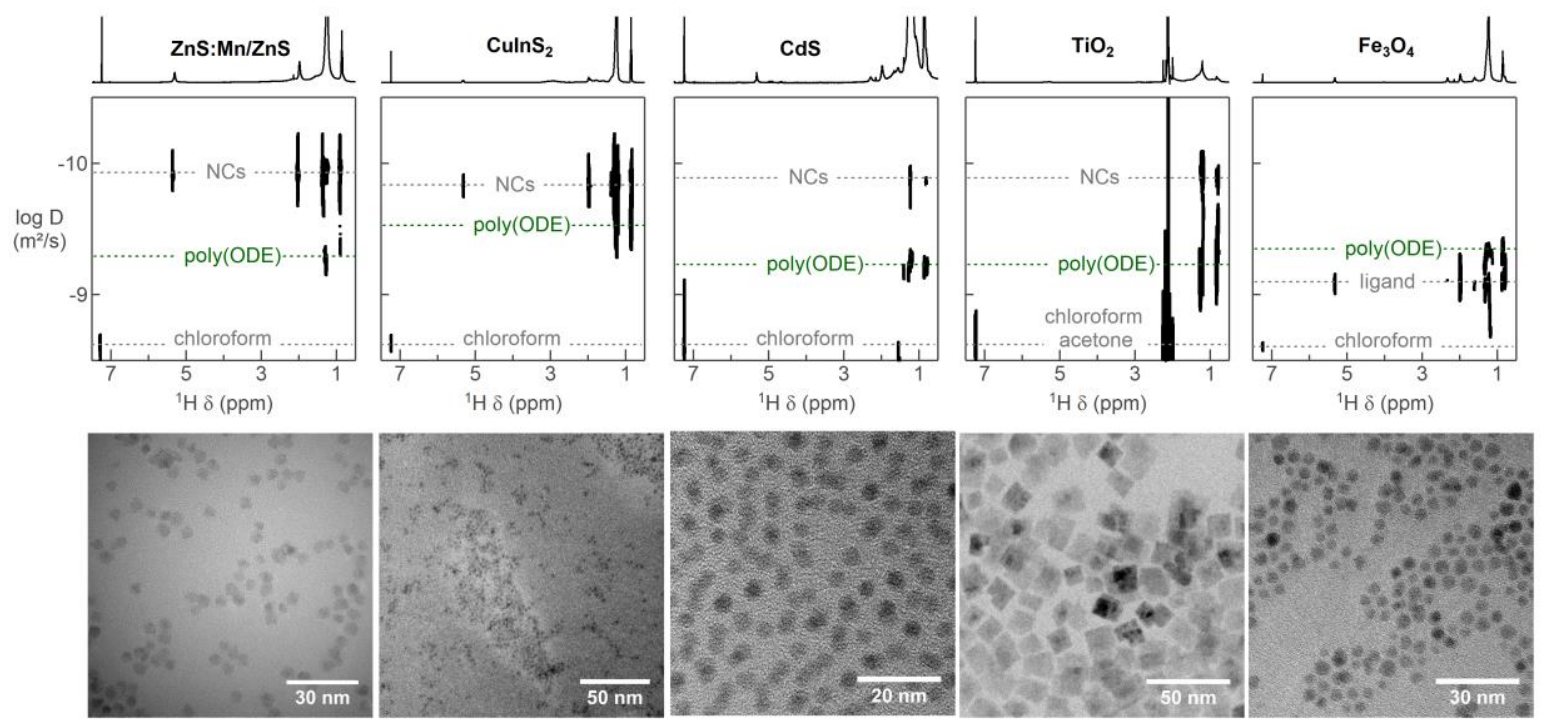

Figure 2. 2D DOSY NMR spectra and TEM images of NCs synthesized in ODE; ZnS:Mn/ZnS (oleate and oleylamine capped), $\mathrm{CuInS}_{2}$ (oleate capped), $\mathrm{CdS}$ (oleate capped), $\mathrm{TiO}_{2}$ (oleylamine capped), and $\mathrm{Fe}_{3} \mathrm{O}_{4}$ (oleate capped). The presence of acetone in the $\mathrm{TiO}_{2} \mathrm{NCs}$ sample is due to the NMR sample procedure (see experimental section). Since the $\mathrm{Fe}_{3} \mathrm{O}_{4} \mathrm{NCs}$ are magnetic, the DOSY measurement is performed after a ligand stripping.

To simply avoid the polymerization of ODE, we explored the substitution of ODE by alternative, saturated solvents. The most obvious candidate is $n$-octadecane, which has a similar boiling point $\left(317^{\circ} \mathrm{C}\right)$. However, $n$-octadecane is a solid at room temperature $\left(\mathrm{T}_{\mathrm{m}}=26-29^{\circ} \mathrm{C}\right)$, which is inconvenient and especially problematic in procedures involving syringe pump addition of stock solutions. More convenient (i.e., liquid at room temperature) solvents are $n$ hexadecane $\left(\mathrm{T}_{\mathrm{b}}=287^{\circ} \mathrm{C}\right)$ or $2,6,10,15,19,23$-hexamethyltetracosane (also known as squalane, $\mathrm{T}_{\mathrm{b}}=350{ }^{\circ} \mathrm{C}$ ). If the solvent is merely a heat transfer medium and does not partake in the reaction, it should be straightforward to substitute ODE. Indeed, for the $\mathrm{CuInS}_{2}, \mathrm{CdS}$, and $\mathrm{TiO}_{2}$ NC syntheses in $n$-hexadecane and for the $\mathrm{Fe}_{3} \mathrm{O}_{4} \mathrm{NC}$ synthesis in $n$-octadecane we observe no obvious differences in NC morphology, size and dispersity (Figure 3). DOSY NMR of those 
four cases confirms that poly(ODE) is absent (Figure 3) and the nanocrystal dispersion is thus pure. Unfortunately, the synthesis of $\mathrm{ZnS}: \mathrm{Mn} / \mathrm{ZnS}$ core/shell NCs is not controlled in $n$ hexadecane and results in highly polydisperse NCs (Figure 3 left). In this type of synthesis, the terminal alkene group of ODE is responsible for the controlled reduction of elemental sulfur. ${ }^{50}$ Therefore, ODE is a necessary component of the synthesis and the formation of poly(ODE) cannot be avoided without compromising the NC quality.

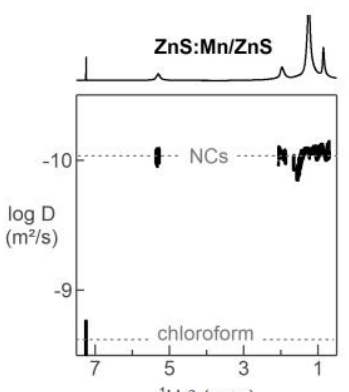

${ }^{1} \mathrm{H} \delta(\mathrm{ppm})$

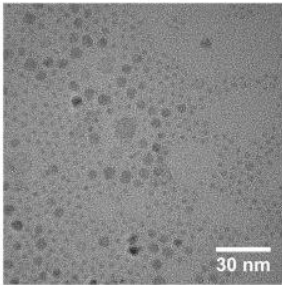

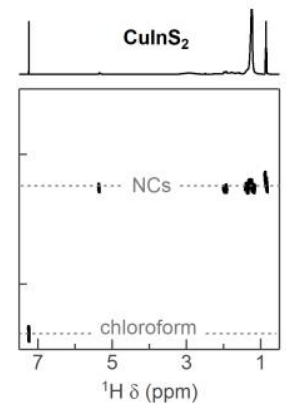

${ }^{1} \mathrm{H} \delta(\mathrm{ppm})$

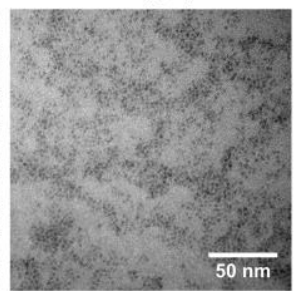

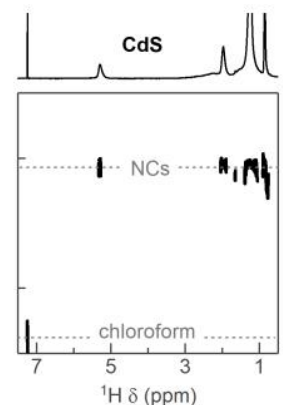

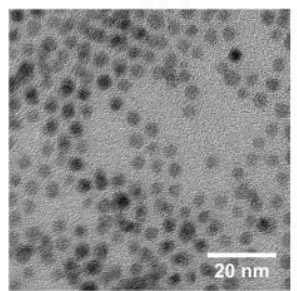

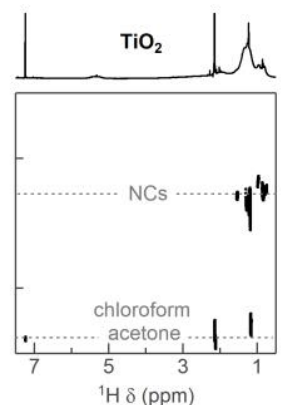
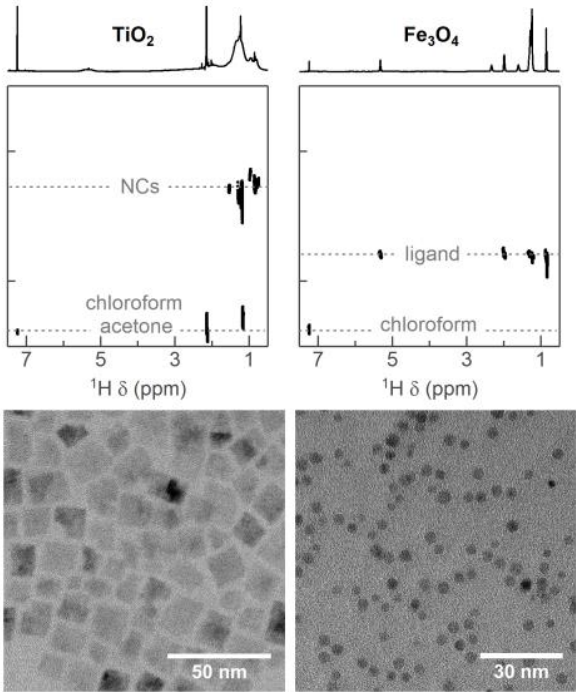

Figure 3. 2D DOSY NMR spectra and TEM images of NCs synthesized in $n$-hexadecane (ZnS:Mn/ZnS core/shell, $\mathrm{CuInS}_{2}, \mathrm{CdS}$, and $\left.\mathrm{TiO}_{2} \mathrm{NCs}\right)$ or $n$-octadecane $\left(\mathrm{Fe}_{3} \mathrm{O}_{4} \mathrm{NCs}\right)$, evidencing the absence of poly(ODE). Since the $\mathrm{Fe}_{3} \mathrm{O}_{4}$ NCs are magnetic, the DOSY measurement is performed after a ligand stripping. All DOSY measurements are performed in a similar NC concentration as in Figure 2.

Nanocrystal syntheses are complex and it is clear that changing the solvent can have significant effects on the NC product. On the other hand, poly(ODE) hampers charge transfer in solid films of $\mathrm{NCs}^{63}$, distorts NMR spectra (thus hampering surface chemistry studies), and generally decreases sample purity. Since poly(ODE) was observed in samples that were purified via the classical precipitation-redispersion cycles (Figure 2), it is desirable to develop alternative purification procedures. In literature, subtle 'tricks' and delicate procedures are reported, giving nanocrystal purification the aura of art instead of science. ${ }^{21-22,} 64$ While size exclusion 
chromatography has been proposed as an interesting new purification technique, ${ }^{64-65}$ we did not succeed in separating the poly(ODE) from ligand capped nanocrystals (Figure S4).

The difficulty in obtaining pure NCs lies with the comparable size and polarity of poly(ODE) and ligand-capped-NCs. While the size cannot be changed, the polarity can be adjust by modification of the surface. We thus exchange the native hydrophobic oleate ligands for the slightly more polar ligand 1: (6-[2-[2-(2-methoxy-ethoxy)-ethoxy]-ethoxy]hexyl) phosphonic acid (Figure 4A). Ligands with multiple ethylene glycol moieties are highly versatile since they provide colloidal stability in a variety of solvents with different polarity, excluding hexanes. ${ }^{17}$, 66-68 Since poly(ODE) is highly soluble in hexanes, this provides a pathway to separate poly(ODE) from the NCs.

To illustrate the procedure, we functionalize the surface of CdS NCs, synthesized in ODE, with 1 (Figure 4A). The as-synthesized CdS NCs are capped with oleate ligands, as evidenced by ${ }^{1} \mathrm{H}$ NMR (Figure 4B). We infer from the high intensity in the aliphatic region of the NMR spectrum $(0.5-1.5 \mathrm{ppm})$ that a substantial amount of poly(ODE) is present. To exchange the oleates for $\mathbf{1}$, we only added a slight excess of $\mathbf{1}$ because phosphonates have a stronger binding affinity to the NC surface. ${ }^{67,}$ 69-70 Subsequently, the NCs are precipitated by hexanes and redispersed in chloroform. The supernatant containing poly(ODE) is discarded. The ${ }^{1} \mathrm{H}$ NMR spectrum of the NCs confirms a successful purification since only the bound (broadened) resonances of $\mathbf{1}$ are observed and the aliphatic signals of poly(ODE) are absent (Figure 4B). The purity of the sample is further confirmed by 2D DOSY NMR which shows only 1 set of resonances with a low diffusion coefficient, corresponding to bound $\mathbf{1}$ (Figure 4C). This is also reflected in the single exponential fit of the DOSY decay curve for the region from 1.7 to $1.2 \mathrm{ppm}$ (Figure 4D). 

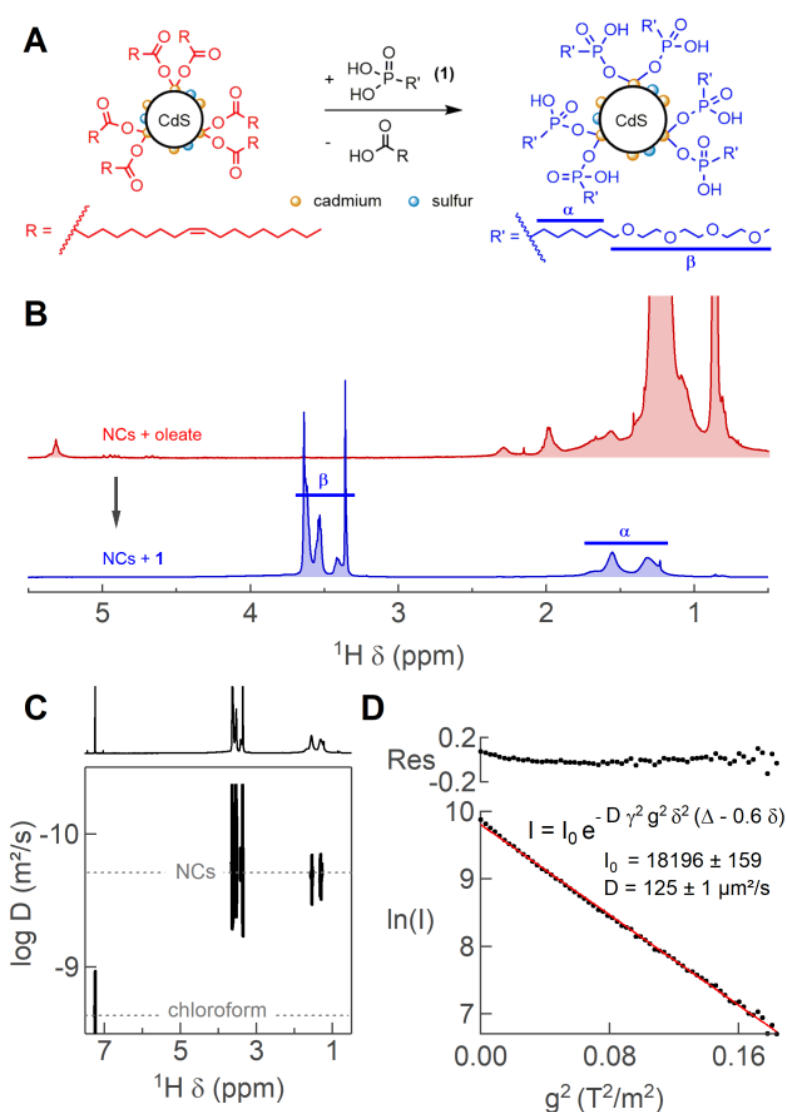

D

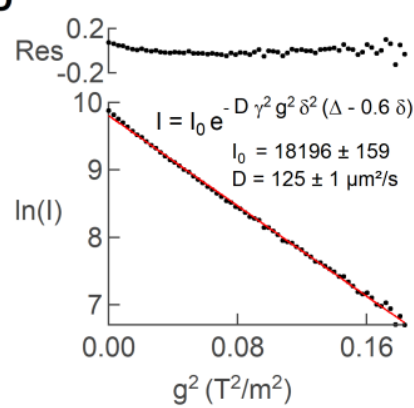

Figure 4. (A) Ligand exchange of oleate for 1 on CdS NCs. (B) The corresponding ${ }^{1} \mathrm{H}$ NMR spectra of CdS NCs capped with oleate and with poly(ODE) present, purified CdS NCs after ligand exchange with $\mathbf{1}$ and a reference spectrum of 1, all in $\mathrm{CDCl}_{3}$. (C) 2D DOSY NMR spectrum shows only the presence of the purified CdS NCs functionalized with ligand 1. (D) The analysis of the DOSY decay curve of the region from 1.7 to $1.2 \mathrm{ppm}$ confirms the presence of only one component, i.e., the purified NCs.

The same procedure can be applied to $\mathrm{ZnS}: \mathrm{Mn} / \mathrm{ZnS}$ NCs but the surface chemistry of the assynthesized NCs is more complex (Figure S6). By stripping the surface ligands with a strong acid, we determined a relative surface composition of $81 \%$ oleate and $19 \%$ oleylamine. ${ }^{61-62} \mathrm{We}$ infer that oleate binds as an X-type ligand while oleylamine binds as an L-type ligand. ${ }^{7}$ This complicates ligand exchange because upon addition of $\mathbf{1}$, both an $\mathrm{X}$-for-X ligand exchange with oleate occurs and an ion pair is formed with oleylamine. Nevertheless, the functionalized NCs can be precipitated with hexanes and redispersed in chloroform. In the ${ }^{31} \mathrm{P}$ NMR spectrum, a broadened resonance at $30 \mathrm{ppm}(\mathrm{FWHM}=1113.51 \mathrm{~Hz})$ is observed, which is assigned to bound X-type phosphonate. The smaller and sharper resonance at $35 \mathrm{ppm}(\mathrm{FWHM}=366.45 \mathrm{~Hz})$ is assigned to the ion pair of $\mathbf{1}$ and oleylamine. It is not surprising that ion pairs are still present 
given their low solubility in hexanes and their reasonably high affinity for the nanocrystal surface. ${ }^{71-72}$ Finally, the ${ }^{1} \mathrm{H}$ NMR spectrum confirms these conclusions as the spectrum is dominated by the resonances of $\mathbf{1}$, but the resonances of oleylamine remain present. As hypothesized, this procedure allowed for the purification of the NCs from the poly(ODE) produced during the synthesis.

In conclusion, $\mathrm{ODE}$ spontaneously polymerizes at temperatures above $120^{\circ} \mathrm{C}$ and we have identified its presence in established syntheses of five types of nanocrystals, i.e., $\mathrm{ZnS}: \mathrm{Mn} / \mathrm{ZnS}$, $\mathrm{CuInS}_{2}, \mathrm{CdS}, \mathrm{TiO}_{2}$, and $\mathrm{Fe}_{3} \mathrm{O}_{4}$ NCs. Standard purification procedures were unable to separate the poly(ODE) impurity from the NC product. While the use of saturated solvents can prevent poly(ODE) from forming, we also developed a surface modification strategy to increase to polarity of the NCs. As such, the nonpolar poly(ODE) could be readily separated from the polar NCs based on solubility differences. In general, the contamination of NC products by poly(ODE) is a problem for NC-based applications. Here we have provided the tools to design improved synthetic protocols towards pure nanocrystals.

\section{Acknowledgements}

The authors acknowledge the FWO Vlaanderen, Special Research Fund/Concerted Research Actions project (BOF2015/GOA/007), Ghent University, Columbia University, Fulbright, the Belgian American Education Foundation and the COMPASS project (H2020-MSCA-RISE2015-691185) for financial support. The authors thank Bernhard De Meyer and prof Filip Du Prez for the SEC analysis.

\section{Associated Content}

\section{Supporting information}

The Supporting Information is available free of charge on the ACS Publications website at DOI: 
Experimental procedures, techniques used to characterize, additional ${ }^{1} \mathrm{H}$ NMR and SEC spectra of poly(ODE), surface chemistry characterization, and rational ligand exchange of ZnS:Mn/ZnS core/shell NCs.

\section{Author Information}

\section{Corresponding Author}

*E-mail: Jonathan.DeRoo@unibas.ch

\section{ORCID}

Evert Dhaene: 0000-0002-1604-0408

Ellie Bennett: 0000-0003-2714-2773

Jonas Billet: 0000-0002-1068-5096

Isabel Van Driessche: 0000-0001-5253-3325

Jonathan De Roo: 0000-0002-1264-9312

\section{Notes}

The authors declare no competing financial interest.

\section{References}

1. Llordés, A.; Garcia, G.; Gazquez, J.; Milliron, D. J., Tunable near-infrared and visiblelight transmittance in nanocrystal-in-glass composites. Nature 2013, 500, 323.

2. Wang, Y.; Fedin, I.; Zhang, H.; Talapin, D. V., Direct optical lithography of functional inorganic nanomaterials. Science 2017, 357 (6349), 385-388.

3. Reiss, P.; Carrière, M.; Lincheneau, C.; Vaure, L.; Tamang, S., Synthesis of Semiconductor Nanocrystals, Focusing on Nontoxic and Earth-Abundant Materials. Chem. Rev. 2016, 116 (18), 10731-10819. 
4. Ibáñez, M.; Luo, Z.; Genç, A.; Piveteau, L.; Ortega, S.; Cadavid, D.; Dobrozhan, O.; Liu, Y.; Nachtegaal, M.; Zebarjadi, M.; Arbiol, J.; Kovalenko, M. V.; Cabot, A., Highperformance thermoelectric nanocomposites from nanocrystal building blocks. Nat. Commun. 2016, 7, 10766.

5. Yin, Y.; Alivisatos, A. P., Colloidal nanocrystal synthesis and the organic-inorganic interface. Nature 2005, 437 (7059), 664-670.

6. Kovalenko, M. V.; Manna, L.; Cabot, A.; Hens, Z.; Talapin, D. V.; Kagan, C. R.; Klimov, V. I.; Rogach, A. L.; Reiss, P.; Milliron, D. J.; Guyot-Sionnnest, P.; Konstantatos, G.; Parak, W. J.; Hyeon, T.; Korgel, B. A.; Murray, C. B.; Heiss, W., Prospects of nanoscience with nanocrystals. ACS Nano 2015, 9 (2), 1012-57.

7. De Roo, J.; De Keukeleere, K.; Hens, Z.; Van Driessche, I., From ligands to binding motifs and beyond; the enhanced versatility of nanocrystal surfaces. Dalton Trans. 2016, 45 (34), 13277-13283.

8. Boles, M. A.; Ling, D.; Hyeon, T.; Talapin, D. V., The surface science of nanocrystals. Nat. Mater. 2016, 15, 141.

9. De Roo, J.; Van Driessche, I.; Martins, J. C.; Hens, Z., Colloidal metal oxide nanocrystal catalysis by sustained chemically driven ligand displacement. Nat. Mater. 2016, 15, 517.

10. Ibáñez, M.; Korkosz, R. J.; Luo, Z.; Riba, P.; Cadavid, D.; Ortega, S.; Cabot, A.; Kanatzidis, M. G., Electron Doping in Bottom-Up Engineered Thermoelectric Nanomaterials through HCl-Mediated Ligand Displacement. J. Am. Chem. Soc. 2015, 137 (12), 4046-4049.

11. Lan, X.; Voznyy, O.; García de Arquer, F. P.; Liu, M.; Xu, J.; Proppe, A. H.; Walters, G.; Fan, F.; Tan, H.; Liu, M.; Yang, Z.; Hoogland, S.; Sargent, E. H., 10.6\% Certified Colloidal Quantum Dot Solar Cells via Solvent-Polarity-Engineered Halide Passivation. Nano Lett. 2016, 16 (7), 4630-4634.

12. Houtepen, A. J.; Hens, Z.; Owen, J. S.; Infante, I., On the Origin of Surface Traps in Colloidal II-VI Semiconductor Nanocrystals. Chem. Mater. 2017, 29 (2), 752-761.

13. Rijckaert, H.; Pollefeyt, G.; Sieger, M.; Hänisch, J.; Bennewitz, J.; De Keukeleere, K.; De Roo, J.; Hühne, R.; Bäcker, M.; Paturi, P.; Huhtinen, H.; Hemgesberg, M.; Van Driessche, I., Optimizing Nanocomposites through Nanocrystal Surface Chemistry: Superconducting YBa2Cu3O7 Thin Films via Low-Fluorine Metal Organic Deposition and Preformed Metal Oxide Nanocrystals. Chem. Mater. 2017, 29 (14), 6104-6113.

14. Hendricks, M. P.; Campos, M. P.; Cleveland, G. T.; Jen-La Plante, I.; Owen, J. S., A tunable library of substituted thiourea precursors to metal sulfide nanocrystals. Science 2015, 348 (6240), 1226-1230.

15. Wang, F.; Tang, R.; Buhro, W. E., The Trouble with TOPO; Identification of Adventitious Impurities Beneficial to the Growth of Cadmium Selenide Quantum Dots, Rods, and Wires. Nano Lett. 2008, 8 (10), 3521-3524.

16. Wang, F.; Tang, R.; Kao, J. L. F.; Dingman, S. D.; Buhro, W. E., Spectroscopic Identification of Tri-n-octylphosphine Oxide (TOPO) Impurities and Elucidation of Their Roles in Cadmium Selenide Quantum-Wire Growth. J. Am. Chem. Soc. 2009, 131 (13), 4983-4994.

17. Owen, J. S.; Park, J.; Trudeau, P.-E.; Alivisatos, A. P., Reaction Chemistry and Ligand Exchange at Cadmium-Selenide Nanocrystal Surfaces. J. Am. Chem. Soc. 2008, 130 (37), 12279-12281.

18. De Keukeleere, K.; Coucke, S.; De Canck, E.; Van Der Voort, P.; Delpech, F.; Coppel, Y.; Hens, Z.; Van Driessche, I.; Owen, J. S.; De Roo, J., Stabilization of Colloidal Ti, Zr, and Hf Oxide Nanocrystals by Protonated Tri-n-octylphosphine Oxide (TOPO) and Its Decomposition Products. Chem. Mater. 2017, 29 (23), 10233-10242.

19. Srivastava, B. B.; Jana, S.; Karan, N. S.; Paria, S.; Jana, N. R.; Sarma, D. D.; Pradhan, N., Highly Luminescent Mn-Doped ZnS Nanocrystals: Gram-Scale Synthesis. J. Phys. Chem. Lett. 2010, 1 (9), 1454-1458. 
20. Chen, B.; Zhong, H.; Zhang, W.; Tan, Z. a.; Li, Y.; Yu, C.; Zhai, T.; Bando, Y.; Yang, S.; Zou, B., Highly Emissive and Color-Tunable CuInS2-Based Colloidal Semiconductor Nanocrystals: Off-Stoichiometry Effects and Improved Electroluminescence Performance. Adv. Funct. Mater. 2012, 22 (10), 2081-2088.

21. Hamachi, L. S.; Jen-La Plante, I.; Coryell, A. C.; De Roo, J.; Owen, J. S., Kinetic Control over CdS Nanocrystal Nucleation Using a Library of Thiocarbonates, Thiocarbamates, and Thioureas. Chem. Mater. 2017, 29 (20), 8711-8719.

22. Gordon, T. R.; Cargnello, M.; Paik, T.; Mangolini, F.; Weber, R. T.; Fornasiero, P.; Murray, C. B., Nonaqueous Synthesis of TiO2 Nanocrystals Using TiF4 to Engineer Morphology, Oxygen Vacancy Concentration, and Photocatalytic Activity. J. Am. Chem. Soc. 2012, 134 (15), 6751-6761.

23. Jana, N. R.; Chen, Y.; Peng, X., Size- and Shape-Controlled Magnetic (Cr, Mn, Fe, Co, Ni) Oxide Nanocrystals via a Simple and General Approach. Chem. Mater. 2004, 16 (20), 39313935.

24. Puglisi, A.; Mondini, S.; Cenedese, S.; Ferretti, A. M.; Santo, N.; Ponti, A., Monodisperse Octahedral $\alpha-\mathrm{MnS}$ and MnO Nanoparticles by the Decomposition of Manganese Oleate in the Presence of Sulfur. Chem. Mater. 2010, 22 (9), 2804-2813.

25. Hsu, S.-W.; Ngo, C.; Bryks, W.; Tao, A. R., Shape Focusing During the Anisotropic Growth of CuS Triangular Nanoprisms. Chem. Mater. 2015, 27 (14), 4957-4963.

26. Wang, Y.; Hu, Y.; Zhang, Q.; Ge, J.; Lu, Z.; Hou, Y.; Yin, Y., One-Pot Synthesis and Optical Property of Copper(I) Sulfide Nanodisks. Inorg. Chem. 2010, 49 (14), 6601-6608.

27. Du, Y.; Xu, B.; Fu, T.; Cai, M.; Li, F.; Zhang, Y.; Wang, Q., Near-Infrared Photoluminescent Ag2S Quantum Dots from a Single Source Precursor. J. Am. Chem. Soc. 2010, 132 (5), 1470-1471.

28. Wang, D.-S.; Zheng, W.; Hao, C.-H.; Peng, Q.; Li, Y.-D., A Synthetic Method for Transition-Metal Chalcogenide Nanocrystals. Chem.: Eur. J. 2009, 15 (8), 1870-1875.

29. Sahu, A.; Qi, L.; Kang, M. S.; Deng, D.; Norris, D. J., Facile Synthesis of Silver Chalcogenide (Ag2E; $\mathrm{E}=\mathrm{Se}, \mathrm{S}, \mathrm{Te})$ Semiconductor Nanocrystals. J. Am. Chem. Soc. 2011, 133 (17), 6509-6512.

30. Liu, X.; Li, Y.; Zhou, B.; Wang, X.; Cartwright, A. N.; Swihart, M. T., Shape-Controlled Synthesis of SnE $(\mathrm{E}=\mathrm{S}, \mathrm{Se})$ Semiconductor Nanocrystals for Optoelectronics. Chem. Mater. 2014, 26 (11), 3515-3521.

31. Bang, J.; Park, J.; Lee, J. H.; Won, N.; Nam, J.; Lim, J.; Chang, B. Y.; Lee, H. J.; Chon, B.; Shin, J.; Park, J. B.; Choi, J. H.; Cho, K.; Park, S. M.; Joo, T.; Kim, S., ZnTe/ZnSe (Core/Shell) Type-II Quantum Dots: Their Optical and Photovoltaic Properties. Chem. Mater. 2010, 22 (1), 233-240.

32. Lee, S. H.; Kim, Y. J.; Park, J., Shape Evolution of ZnTe Nanocrystals: Nanoflowers, Nanodots, and Nanorods. Chem. Mater. 2007, 19 (19), 4670-4675.

33. Kovalenko, M. V.; Heiss, W.; Shevchenko, E. V.; Lee, J.-S.; Schwinghammer, H.; Alivisatos, A. P.; Talapin, D. V., SnTe Nanocrystals: A New Example of Narrow-Gap Semiconductor Quantum Dots. J. Am. Chem. Soc. 2007, 129 (37), 11354-11355.

34. Jasieniak, J.; Bullen, C.; van Embden, J.; Mulvaney, P., Phosphine-Free Synthesis of CdSe Nanocrystals. J. Phys. Chem. B 2005, 109 (44), 20665-20668.

35. Zhong, X.; Feng, Y.; Knoll, W.; Han, M., Alloyed ZnxCd1-xS Nanocrystals with Highly Narrow Luminescence Spectral Width. J. Am. Chem. Soc. 2003, 125 (44), 13559-13563.

36. Bae, W. K.; Nam, M. K.; Char, K.; Lee, S., Gram-Scale One-Pot Synthesis of Highly Luminescent Blue Emitting Cd1-xZnxS/ZnS Nanocrystals. Chem. Mater. 2008, 20 (16), 53075313.

37. Li, Z.; Ji, Y.; Xie, R.; Grisham, S. Y.; Peng, X., Correlation of CdS Nanocrystal Formation with Elemental Sulfur Activation and Its Implication in Synthetic Development. $J$. Am. Chem. Soc. 2011, 133 (43), 17248-17256. 
38. Cao, Y. C.; Wang, J., One-Pot Synthesis of High-Quality Zinc-Blende CdS Nanocrystals. J. Am. Chem. Soc. 2004, 126 (44), 14336-14337.

39. Ouyang, J.; Kuijper, J.; Brot, S.; Kingston, D.; Wu, X.; Leek, D. M.; Hu, M. Z.; Ripmeester, J. A.; Yu, K., Photoluminescent Colloidal CdS Nanocrystals with High Quality via Noninjection One-Pot Synthesis in 1-Octadecene. J. Phys. Chem. C 2009, 113 (18), 7579-7593. 40. Yang, Y. A.; Wu, H.; Williams, K. R.; Cao, Y. C., Synthesis of CdSe and CdTe Nanocrystals without Precursor Injection. Angew. Chem. 2005, 44 (41), 6712-6715.

41. Liu, L.; Zhuang, Z.; Xie, T.; Wang, Y.-G.; Li, J.; Peng, Q.; Li, Y., Shape Control of CdSe Nanocrystals with Zinc Blende Structure. J. Am. Chem. Soc. 2009, 131 (45), 1642316429 .

42. Ithurria, S.; Bousquet, G.; Dubertret, B., Continuous Transition from 3D to 1D Confinement Observed during the Formation of CdSe Nanoplatelets. J. Am. Chem. Soc. 2011, 133 (9), 3070-3077.

43. Bullen, C.; van Embden, J.; Jasieniak, J.; Cosgriff, J. E.; Mulder, R. J.; Rizzardo, E.; Gu, M.; Raston, C. L., High Activity Phosphine-Free Selenium Precursor Solution for Semiconductor Nanocrystal Growth. Chem. Mater. 2010, 22 (14), 4135-4143.

44. De Nolf, K.; Capek, R. K.; Abe, S.; Sluydts, M.; Jang, Y.; Martins, J. C.; Cottenier, S.; Lifshitz, E.; Hens, Z., Controlling the Size of Hot Injection Made Nanocrystals by Manipulating the Diffusion Coefficient of the Solute. J. Am. Chem. Soc. 2015, 137 (7), 2495-2505.

45. Della Gaspera, E.; Chesman, A. S. R.; van Embden, J.; Jasieniak, J. J., Non-injection Synthesis of Doped Zinc Oxide Plasmonic Nanocrystals. ACS Nano 2014, 8 (9), 9154-9163.

46. Liu, H.; Owen, J. S.; Alivisatos, A. P., Mechanistic Study of Precursor Evolution in Colloidal Group II-VI Semiconductor Nanocrystal Synthesis. J. Am. Chem. Soc. 2007, 129 (2), 305-312.

47. Park, J.; An, K.; Hwang, Y.; Park, J.-G.; Noh, H.-J.; Kim, J.-Y.; Park, J.-H.; Hwang, N.M.; Hyeon, T., Ultra-large-scale syntheses of monodisperse nanocrystals. Nat. Mater. 2004, 3, 891.

48. Wu, L.; Willis, J. J.; McKay, I. S.; Diroll, B. T.; Qin, J.; Cargnello, M.; Tassone, C. J., High-temperature crystallization of nanocrystals into three-dimensional superlattices. Nature 2017, 548, 197.

49. Li, L.; Daou, T. J.; Texier, I.; Kim Chi, T. T.; Liem, N. Q.; Reiss, P., Highly Luminescent CuInS2/ZnS Core/Shell Nanocrystals: Cadmium-Free Quantum Dots for In Vivo Imaging. Chem. Mater. 2009, 21 (12), 2422-2429.

50. García-Rodríguez, R.; Hendricks, M. P.; Cossairt, B. M.; Liu, H.; Owen, J. S., Conversion Reactions of Cadmium Chalcogenide Nanocrystal Precursors. Chem. Mater. 2013, 25 (8), 1233-1249.

51. Yordanov, G. G.; Yoshimura, H.; Dushkin, C. D., Phosphine-free synthesis of metal chalcogenide quantum dots by means of in situ-generated hydrogen chalcogenides. Colloid Polym. Sci. 2008, 286, 813-817.

52. Chen, O.; Chen, X.; Yang, Y.; Lynch, J.; Wu, H.; Zhuang, J.; Cao, Y. C., Synthesis of Metal-Selenide Nanocrystals Using Selenium Dioxide as the Selenium Precursor. Angew. Chem. 2008, 47 (45), 8638-8641.

53. Quijada, R.; Guevara, J. L.; Yazdani-Pedram, M.; Galland, G. B.; Ribeiro, D., Study of the polymerization of 1-octadecene with different rnetallocene catalysts. Polym. Bull. 2002, 49 (4), 273-280.

54. Grumel, V.; Brüll, R.; Pasch, H.; Raubenheimer, H. G.; Sanderson, R.; Wahner, U. M., Homopolymerization of Higher 1-Olefins with Metallocene/MAO Catalysts. Macromol. Mater. Eng. 2001, 286 (8), 480-487.

55. Brüll, R.; Kgosane, D.; Neveling, A.; Pasch, H.; Raubenheimer, H. G.; Sanderson, R.; Wahner, U. M., Synthesis and properties of poly-1-olefins. Macromol. Symp. 2001, 165 (1), 11-18. 
56. Zapata, P. A.; Palza, H.; Cruz, L. S.; Lieberwirth, I.; Catalina, F.; Corrales, T.; Rabagliati, F. M., Polyethylene and poly(ethylene-co-1-octadecene) composites with TiO2 based nanoparticles by metallocenic "in situ" polymerization. Polymer 2013, 54 (11), 26902698.

57. Aubrey, D. W.; Barnatt, A., Polymerization of n-octadecene-1 with catalysts derived from titanium tetrachloride and triethylaluminum. J. Polym. Sci. A 1966, 4 (7), 1709-1726.

58. Srivastava, V.; Liu, W.; Janke, E. M.; Kamysbayev, V.; Filatov, A. S.; Sun, C.-J.; Lee, B.; Rajh, T.; Schaller, R. D.; Talapin, D. V., Understanding and Curing Structural Defects in Colloidal GaAs Nanocrystals. Nano Lett. 2017, 17 (3), 2094-2101.

59. Dainton, F. S.; Ivin, K. J., Reversibility of the Propagation Reaction in Polymerization Processes and its Manifestation in the Phenomenon of a 'Ceiling Temperature\&\#39. Nature 1948, $162,705$.

60. Rosenboom, J.-G.; De Roo, J.; Storti, G.; Morbidelli, M., Diffusion (DOSY) 1H NMR as an Alternative Method for Molecular Weight Determination of Poly(ethylene furanoate) (PEF) Polyesters. Macromol. Chem. Phys. 2016, 218 (1), 1600436.

61. Berestok, T.; Guardia, P.; Blanco, J.; Nafria, R.; Torruella, P.; López-Conesa, L.; Estradé, S.; Ibáñez, M.; de Roo, J.; Luo, Z.; Cadavid, D.; Martins, J. C.; Kovalenko, M. V.; Peiró, F.; Cabot, A., Tuning Branching in Ceria Nanocrystals. Chem. Mater. 2017, 29 (10), 4418-4424.

62. Liu, Y.; Cadavid, D.; Ibáñez, M.; De Roo, J.; Ortega, S.; Dobrozhan, O.; V. Kovalenko, M.; Cabot, A., Colloidal AgSbSe2 nanocrystals: surface analysis, electronic doping and processing into thermoelectric nanomaterials. J. Mater. Chem. C 2016, 4 (21), 4756-4762.

63. Tisdale, W. A.; Williams, K. J.; Timp, B. A.; Norris, D. J.; Aydil, E. S.; Zhu, X.-Y., Hot-Electron Transfer from Semiconductor Nanocrystals. Science 2010, 328 (5985), 15431547.

64. Shen, Y.; Gee, M. Y.; Greytak, A. B., Purification technologies for colloidal nanocrystals. ChemComm 2017, 53 (5), 827-841.

65. Shen, Y.; Gee, M. Y.; Tan, R.; Pellechia, P. J.; Greytak, A. B., Purification of Quantum Dots by Gel Permeation Chromatography and the Effect of Excess Ligands on Shell Growth and Ligand Exchange. Chem. Mater. 2013, 25 (14), 2838-2848.

66. De Roo, J.; Yazdani, N.; Drijvers, E.; Lauria, A.; Maes, J.; Owen, J. S.; Van Driessche, I.; Niederberger, M.; Wood, V.; Martins, J. C.; Infante, I.; Hens, Z., Probing Solvent-Ligand Interactions in Colloidal Nanocrystals by the NMR Line Broadening. Chem. Mater. 2018, 30 (15), 5485-5492.

67. De Roo, J.; Zhou, Z.; Wang, J.; Deblock, L.; Crosby, A. J.; Owen, J. S.; Nonnenmann, S. S., Synthesis of Phosphonic Acid Ligands for Nanocrystal Surface Functionalization and Solution Processed Memristors. Chem. Mater. 2018, 30 (21), 8034-8039.

68. Rechberger, F.; Heiligtag, F. J.; Süess, M. J.; Niederberger, M., Assembly of BaTiO3 Nanocrystals into Macroscopic Aerogel Monoliths with High Surface Area. Angew. Chem. 2014, 53 (26), 6823-6826.

69. Gomes, R.; Hassinen, A.; Szczygiel, A.; Zhao, Q.; Vantomme, A.; Martins, J. C.; Hens, Z., Binding of Phosphonic Acids to CdSe Quantum Dots: A Solution NMR Study. J. Phys. Chem. Lett. 2011, 2 (3), 145-152.

70. $\quad$ Knauf, R. R.; Lennox, J. C.; Dempsey, J. L., Quantifying Ligand Exchange Reactions at CdSe Nanocrystal Surfaces. Chem. Mater. 2016, 28 (13), 4762-4770.

71. Chen, P. E.; Anderson, N. C.; Norman, Z. M.; Owen, J. S., Tight Binding of Carboxylate, Phosphonate, and Carbamate Anions to Stoichiometric CdSe Nanocrystals. J. Am. Chem. Soc. 2017, 139 (8), 3227-3236.

72. Billet, J.; Dujardin, W.; De Keukeleere, K.; De Buysser, K.; De Roo, J.; Van Driessche, I., Size Tunable Synthesis and Surface Chemistry of Metastable TiO2-Bronze Nanocrystals. Chem. Mater. 2018, 30 (13), 4298-4306. 


\section{TOC Graphic:}

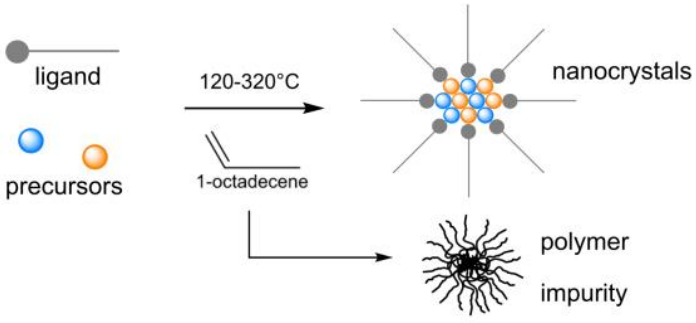

